\title{
Application of Brain Emotional Learning Based Intelligent Controller (BELBIC) to Active Queue Management
}

\author{
Mahdi Jalili-Kharaajoo \\ Young Researchers Club, Azad University, Tehran, Iran \\ mahdijalili@ece.ut.ac.ir
}

\begin{abstract}
In this paper, Brain Emotional Learning Based Intelligent controller (BELBIC) is applied to Active Queue management (AQM). This type of controller is insensitive to noise and variance of the parameters, thus it is suitable to time varying network systems. Simulation results show the robust performance of BELBIC against the disturbance.
\end{abstract}

\section{Introduction}

Active Queue Management (AQM), as one class of packet dropping/marking mechanism in the router queue, has been recently proposed to support the end-to-end congestion control in the Internet [1-3]. AQM highlights the tradeoff between delay and throughput. By keeping the average queue size small, AQM will have the ability to provide greater capacity to accommodate nature-occurring burst without dropping packets, at the same time, reduce the delays seen by flow, this is very particularly important for real-time interactive applications.

Modeling emotions has attracted much attention in recent years, both in cognitive psychology and design of artificial systems [4]. Far from being a negative factor in decision making, emotions have shown to be a strong faculty for making fast satisficing decisions. In this paper, we will adapt a computational model based on the limbic system in the mammalian brain for control engineering applications. We applied the proposed controller (termed BELBIC) [5] for AQM. Our results demonstrate excellent control action, disturbance handling and system parameter robustness for BELBIC.

\section{TCP Flow Control Model}

In [6,7], a nonlinear dynamic model for TCP flow control has been developed based on fluid-flow theory. The state space description of this model can be as follows [1]

$$
\begin{aligned}
& \left\{\begin{array}{l}
\frac{d x_{1}}{d t}=x_{2} \\
\frac{d x_{2}}{d t}=-a_{1}(t) x_{1}-a_{2}(t) x_{2}-b(t)+F(t)
\end{array}\right. \\
& a_{1 \text { min }} \leq a_{1} \leq a_{1 \text { max }}, a_{2 \text { min }} \leq a_{2} \leq a_{2 \text { max }}, 0<b_{\text {min }} \leq b \leq b_{\text {max }}
\end{aligned}
$$


where

$$
\begin{aligned}
& a_{1}(t)=\frac{1}{T_{1}(t) T_{2}(t)}, a_{2}(t)=\frac{T_{1}(t)+T_{2}(t)}{T_{1}(t) T_{2}(t)}, b(t)=\frac{K(t)}{T_{1}(t) T_{2}(t)} \\
& F(t)=\frac{d^{2}}{d t^{2}} q_{o}+\frac{T_{1}(t)+T_{2}(t)}{T_{1}(t) T_{2}(t)} \frac{d}{d t} q_{o}+\frac{1}{T_{1}(t) T_{2}(t)} q_{o}
\end{aligned}
$$

For the purpose of the design of intelligent controller, the varying scope of parameters in TCP/AQM system is assumed as following

$$
N(t): 1-300, T_{p}=0.02 \mathrm{sec}, q_{o}: 0-300 \text { packets, } C(t): 1250-7500 \text { packet } / \mathrm{sec}
$$

Therefore,

$$
a_{2 \min }=3.8501 a_{2 \max }=1250 a_{1 \min }=0.015 a_{1 \max }=60000 b_{\min }=26042, b_{\max }=2812500 .
$$

\section{Brain Emotional Learning Based Intelligent Controller (BELBIC)}

The model of BELBIC is illustrated in Fig. 1 [5]. BELBIC is essentially an action generation mechanism based on sensory inputs and emotional cues. In general, these can be vector valued, although in the benchmark discussed in this paper for the sake of illustration, one sensory input and one emotional signal (stress) have been considered. The emotional learning occurs mainly in amygdala. The learning rule of amygdala is

$$
\Delta G_{a}=k_{1} \cdot \max (0, E C-A)
$$

where $G_{a}$ is the gain in amygdala connection, $k_{1}$ is the learning step in amygdala and $E C$ and $A$ are the values of emotional cue function and amygdala output at each time.

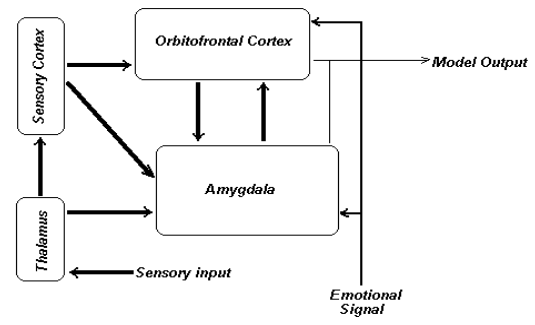

Fig. 1. The abstract structure of BELBIC

Similarly, the learning rule in orbitofrontal cortex is

$$
\Delta G_{o}=k_{2} \cdot(M O-E C)
$$

where $G_{o}$ is the gain in orbitofrontal connection, $k_{2}$ is the learning step in orbitofrontal cortex and $M O$ is the output of the whole model, where it can be calculated as

$$
M O=A-O
$$

in which, $O$ represents the output of orbitofrontal cortex. In fact, by receiving the sensory input $S$, the model calculates the internal signals of amygdala and orbitofrontal cortex by the relations 


$$
A=G_{a} . S, O=G_{o} . S
$$

Since amygdala does not have the capability to unlearn any emotional response that it ever learned, inhibition of any inappropriate response is the duty of orbitofrontal cortex. Controllers based on emotional learning have shown very good robustness and uncertainty handling properties [4,5], while being simple and easily implementable.

The structure of the control circuit we implemented in our study is illustrated in Fig. 2. The functions we used in emotional cue and sensory input blocks are

$$
E C=W_{1} \cdot e+W_{2} \cdot C O, S I=W_{3} \cdot P O+W_{4} \cdot P O
$$

where $E C, C O, S I$ and $P O$ are emotional cue, controller output, sensory input and plant output and the $W_{1}$ through $W_{4}$ are the gains must tuned for designing a satisfactory controller.

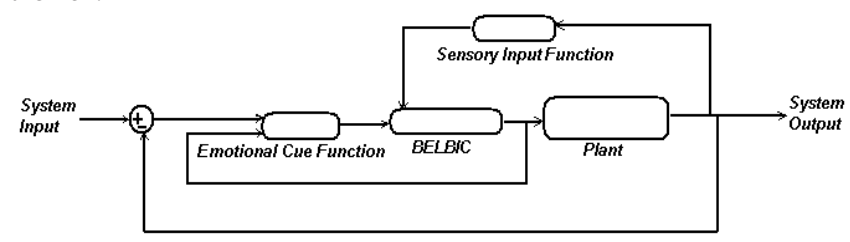

Fig. 2. Control system configuration using

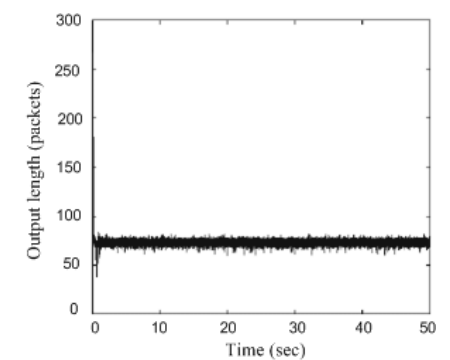

Fig. 3. Queue evaluation (BELBIC)

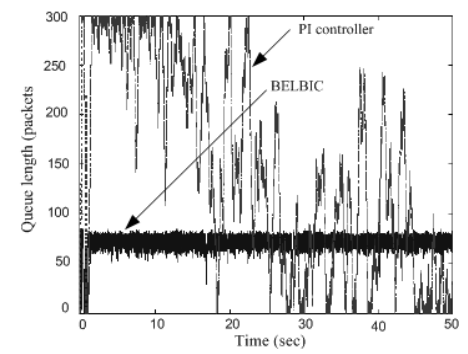

Fig. 5. Queue evaluation (FTP+UDP)

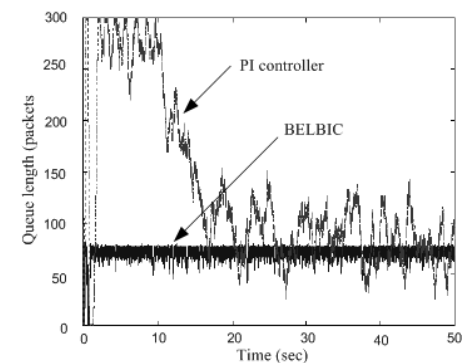

Fig. 4. Queue evaluation (FTP+HTTP)

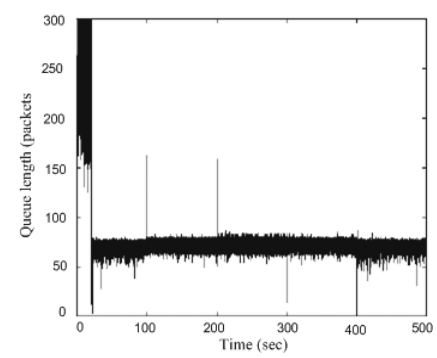

Fig. 6. Queue evaluation using BELBIC

\section{Simulation Results}

The network topology used for simulation is a FTP+UDP+HTTP network, which has been described in details in [1]. We introduced short-lived HTTP flows and non- 
responsive UDP services into the router in order to generate a more realistic scenario, because it is very important for a perfect AQM scheme to achieve full bandwidth utilization in the presence of noise and disturbance introduced by these flows [1].

In the first study, we will use the most general FTP network configuration to testify whether the BELBIC controller can reach the goals of AQM, and freely control the queue length to stabilize at the arbitrary expected value. Let the expected queue length equal to 75 packets, the instantaneous queue length is depicted in Fig. 3. After a very short regulating process, the queue settles down its stable operating point. RED algorithm is unable to accurately control the queue length to the desired value. The queue length varies with network loads.

For FTP+HTTP configuration the evaluation of queue size is shown in Fig. 4 using BELBIC and classical PI controller. As it can be seen, BELBIC has better performance than that of PI one. This comparison is done for FTP+UDP in Fig. 5. Obviously, PI controller is very sensitive to this disturbance, while BELBIC operates in a relatively stable state. The queue fluctuation increases with introducing the UDP flows, but the variance is too much smaller comparing with PI one.

Finally, we evaluate the integrated performance of BELBIC using one relatively real scenario, i.e., the number of active flows is changeable, which has 270 FTP flows, 400 HTTP connections and 30 UDP flows (Fig. 6).

\section{Conclusion}

In this paper, BELBIC wad applied to active queue management. BELBIC is insensitive to system dynamic parameters and is capable of being against disturbance and noise, which is very suitable for the mutable network environment. BELBIC was very responsive, stable and robust, especially for the small reference queue system, but its performance was inferior when active TCP sessions were relatively small.

\section{References}

1. Jalili-Kharaajoo, M., Application of robust fuzzy second order sliding mode control to active queue management, Lecture Notes in Computer Science, 2957, pp.109-119, 2004.

2. Barden, B. et al., Recommendation on queue management and congestion avoidance in the internet, REC2309, April 1998.

3. Firoiu, V. and Borden, M., A study of active queue management for congestion control, Proc. INFOCOM, March 2000.

4. J. Moren, C. Balkenius, A Computational Model of Emotional Learning in The Amygdala, From Animals to Animals: Proc. $6^{\text {th }}$ Int. conf. Simulation of adaptive behavior, Cambridge, MA, 2000.

5. Lucas, C. and Shahmirzadi, D., Introducing Brain Emotional Learning Based Intelligent Controller (BELBIC), to appear in Intelligent Automation and Soft Computing, 2004.

6. Misra, V., Gong, W.B. and Towsley, D., Fluid-based analysis of network of AQM routers supporting TCP flows with an application to RED, Proc. ACM/SIGCOMM, 2000.

7. Hollot, C., Misra, V., Towsley, D. and Gong, W.B., On designing improved controllers for AQM routers supporting TCP flows, Proc. INFOCOM, 2001. 